Stud. Univ. Babeş-Bolyai Math. 64(2019), No. 4, 529-536

DOI: 10.24193/subbmath.2019.4.07

\title{
Statistical $e$-convergence of double sequences on probabilistic normed spaces
}

\author{
Sevda Akdă̆
}

\begin{abstract}
The concept of statistical convergence for double sequences on probabilistic normed spaces was presented by Karakus and Demirci in 2007. The purpose of this paper is to introduce the concept of statistical $e$-convergence for double sequences and study some fundamental properties of statistical $e$-convergence for double sequences on probabilistic normed spaces.
\end{abstract}

Mathematics Subject Classification (2010): 40A05, 40G15, 40B05.

Keywords: Double sequences, t-norm, probabilistic normed spaces, $e$-convergence, statistical $e$-convergence.

\section{Introduction}

Statistical convergence which is a generalization of the notion of ordinary convergence was first introduced by Fast [4] and Steinhaus [21] in 1951. Then several generalizations and applications of this notion have been investigated by various authors [6], [11], [12], [14]. The concept of statistical convergence for double sequences was studied by Mursaleen and Edely [15]. Boos et al ([2], [3]) introduced and investigated the notion of $e$-convergence of double sequences which is essentially weaker than the Pringsheim convergence. Recently, Sever and Talo [19] have generalized the notion of $e$-convergence to statistical $e$-convergence for a double sequence [see also [20]].

The theory of probabilistic normed spaces [5] originated from the concept of statistical metric spaces which was introduced by Menger [13] and further studied by Schweizer and Sklar [17], [18]. It provides an important method of generalizing the deterministic results of normed linear spaces. It has also very useful applications in various fields, e.g., continuity properties [1], topological spaces [5], study of boundedness [7], convergence of random variables [8] etc.

The idea of statistical convergence of single sequences on probabilistic normed spaces was studied by Karakus in [9]. Then, Karakus and Demirci extended the concept of statistical convergence from single to double sequences in [10]. In this paper we introduce and study the concept of statistical $e$-convergence for double sequences on probabilistic normed spaces. 


\section{Background and preliminaries}

First, we recall some notions and basic definitions those will be used in this paper. Throughout this paper, $\mathbb{N}, \mathbb{R}$ respectively denote the sets of positive integers and real numbers whereas $\mathbb{N} \times \mathbb{N}$ denotes the usual product set.

Definition 2.1. [5] A function $g: \mathbb{R} \rightarrow \mathbb{R}_{0}^{+}$is called a distribution function if the following conditions holds:

a) it is non-decreasing,

b) it is left-continuous,

c) $\inf _{t \in \mathbb{R}} g(t)=0$ and $\sup _{t \in \mathbb{R}} g(t)=1$.

The set of all distribution functions will be denoted by $E$.

Definition 2.2. [18] A triangular norm or briefly $t$-norm is a continuous mapping $*:[0,1] \times$ $[0,1] \rightarrow[0,1]$ such that $([0,1], *)$ is an abelian monoid with unit one and $p * q \geq m * n$ if $p \geq m$ and $q \geq n$ for all $m, n, p, q \in[0,1]$.

For example the $*$ operations

$$
m * n=m n, m * n=\min \{m, n\} \text { and } m * n=\max \{m+n-1,0\}
$$

are $t$-norms on $[0,1]$.

Definition 2.3. [18] If $D$ is a real vector space, $\eta$ is a mapping from $D$ into $E$ (for $x \in D$ the distribution function $\eta(x)$ is denoted by $\eta_{x}$ and $\eta_{x}(t)$ is the value of $\eta_{x}$ at $\left.t \in \mathbb{R}\right)$ and $*$ is a $t$-norm satisfying the following conditions :

i) $\eta_{x}(0)=0$,

ii) $\eta_{x}(t)=1$ for all $t>0$ if and only if $x=0$,

iii) $\eta_{\alpha x}(t)=\eta_{x}\left(\frac{t}{|\alpha|}\right)$ for all $\alpha \in \mathbb{R} \backslash\{0\}$ and for all $t>0$,

iv) $\eta_{x+y}(s+t) \geq \eta_{x}(s) * \eta_{y}(t)$ for all $x, y \in D$ and $s, t \in \mathbb{R}_{0}^{+}$,

then $(D, \eta, *)$ is called a probabilistic normed space (briefly, a PNS).

Definition 2.4. Let $(D, \eta, *)$ be a PNS. Then, a sequence $\left(x_{k}\right)$ is said to be convergent to $L \in$ $D$ with respect to the probabilistic norm $\eta$, that is $x_{k} \stackrel{\eta}{\rightarrow} L$ if for every $\varepsilon>0$ and $\lambda \in(0,1)$ there exists a positive integer $k_{0}$ such that $\eta_{x_{k}-L}(\varepsilon)>1-\lambda$ whenever $k \geq k_{0}$. In this case we write $\eta-\lim x_{k}=L$ as $k \rightarrow \infty$.

Remark 2.5. Let $(D,\|\|$.$) be a real normed space and$

$$
\eta_{x}(t)=\frac{t}{t+\|x\|}
$$

where $x \in D$ and $t \geq 0$ (standard $x$-norm induced by $\|\cdot\|$ ). Then we can see that $x_{k} \stackrel{\|\cdot\|}{\rightarrow} x$ if and only if $x_{k} \stackrel{\eta}{\rightarrow} x$.

\section{Statistical $e$-convergence of double sequence on PNS}

In this section we study the concept of statistical $e$-convergence for double sequences in probabilistic normed space. First, we recall the concept of statistical convergence.

Let $K \subseteq \mathbb{N}$. Then the asymptotic (or natural) density of $K$ denoted by $\delta(K)$ is given by

$$
\delta(K):=\lim _{n} \frac{1}{n}|\{k \leq n: k \in K\}|
$$


whenever the limit exists, where the vertical bars denote the cardinality of the enclosed set.

A number sequence $\left(x_{k}\right)$ is said to be statistically convergent to the number $L$ if for each $\varepsilon>0$ the set

$$
K(\varepsilon):=\left\{k \in \mathbb{N}:\left|x_{k}-L\right| \geq \varepsilon\right\}
$$

has asymptotic density zero, i.e.

$$
\lim _{n} \frac{1}{n}\left|\left\{k \leq n:\left|x_{k}-L\right| \geq \varepsilon\right\}\right|=0 .
$$

In this case we write $s t-\lim x_{k}=L$ as $k \rightarrow \infty$.

So we give the concept of statistical convergence of double sequences.

By the convergence of a double sequence we mean the convergence in the Pringsheim sense that is, a double sequence $\left(x_{k l}\right)$ has Pringsheim limit $L$ provided that given $\varepsilon>0$ there exists $N \in \mathbb{N}$ such that $\left|x_{k l}-L\right|<\varepsilon$ wherever $k, l>N[16]$. We write this as $P-\lim _{k, l} x_{k l}=L$. In case of this convergence, the row-index $k$ and column-index $l$ tend independently to infinity.

We can give the analogue of Definition 2.4 for a double sequence as follows:

Definition 3.1. [10] Let $(D, \eta, *)$ be a PNS. Then, a double sequence $\left(x_{k l}\right)$ is said to be convergent to $L \in D$ with respect to the probabilistic norm $\eta$, that is $x_{k l} \stackrel{\eta}{\rightarrow} L$ if for every $\varepsilon>0$ and $\lambda \in(0,1)$ there exists a positive integer $k_{0}$ such that $\eta_{x_{k l}-L}(\varepsilon)>1-\lambda$ whenever $k, l \geq k_{0}$. In this case we write $\eta_{2}-\lim x_{k l}=L$ as $k, l \rightarrow \infty$.

Let $K \subseteq \mathbb{N} \times \mathbb{N}$ and $K(n, m)$ be the numbers of $(i, j)$ in $K$ such that $i \leq n$ and $j \leq m$. Then the two dimensional analog of natural density can be defined as follows:

$$
\underline{\delta_{2}}(K):=\lim _{n, m} \frac{K(n, m)}{n m} .
$$

For example, let $K=\left\{\left(i^{2}, j^{2}\right): i, j \in \mathbb{N}\right\}$. Then the set $K$ has double natural density zero.

Definition 3.2. [15] A double sequence $\left(x_{k l}\right)$ is said to be statistically convergent to a number $\alpha$ if for each $\varepsilon>0$ the set

$$
\left\{(k, l), k \leq n, l \leq m:\left|x_{k l}-\alpha\right| \geq \varepsilon\right\}
$$

has double natural density zero. We write this as $s t_{2}-\lim _{k, l} x_{k l}=\alpha$.

Definition 3.3. [10] Let $(D, \eta, *)$ be a PNS. Then, a double sequence $\left(x_{k l}\right)$ is said to be statistically convergent to $L \in D$ with respect to the probabilistic norm $\eta$ if for every $\varepsilon>0$ and $\lambda \in(0,1)$ the set

$$
\left\{(k, l), k \leq n, l \leq m: \eta_{x_{k l}-\alpha}(\varepsilon) \leq 1-\lambda\right\}
$$

has double natural density zero. In this case we write $s t_{\eta_{2}}-\lim _{k, l} x_{k l}=\alpha$.

Boos, Leiger and Zeller [3] and Boos [2] introduced and investigated the notion of $e$-convergence of double sequences, which is essentially weaker than the Pringsheim convergence as follows:

Definition 3.4. A double sequence $\left(x_{k l}\right)$ is said to be $e$-convergent to a number $\alpha$ if

$$
\forall \varepsilon>0, \exists l_{0} \in \mathbb{N} \forall l \geq l_{0}, \exists k_{l} \in \mathbb{N} \forall k \geq k_{l},\left|x_{k l}-\alpha\right|<\varepsilon .
$$

We write this as $e-\lim _{k, l} x_{k l}=\alpha$. 
In contrast to the Pringsheim notion of convergence, $e$-convergence states that the row-index $k$ depends on the column-index $l$ whenever it tends to infinity.

Recently, Sever and Talo [19] have defined the concept of statistical $e$-convergence for a double sequence as follows:

Definition 3.5. [19] A double sequence $\left(x_{k l}\right)$ is said to be statistically $e$-convergent to a number $\alpha$ if for every $\varepsilon>0$ the set

$$
\left\{l: \delta\left(\left\{k:\left|x_{k l}-\alpha\right| \geq \varepsilon\right\}\right)=0\right\}
$$

has natural density 1 , that is

$$
\delta\left(\left\{l: \delta\left(\left\{k:\left|x_{k l}-\alpha\right| \geq \varepsilon\right\}\right)=0\right\}\right)=1 .
$$

In this case, one writes $s t_{(e)}-\lim _{k, l} x_{k l}=\alpha$.

Now we give the analogue of these definitions with respect to the probabilistic norm $\eta$.

Definition 3.6. Let $(D, \eta, *)$ be a PNS. A double sequence $\left(x_{k l}\right)$ is said to be $e$-convergent to $\alpha \in D$ with respect to the probabilistic norm $\eta$ provided that for every $\varepsilon>0$ and $\lambda \in(0,1)$

$$
\exists l_{0} \in \mathbb{N} \forall l \geq l_{0}, \exists k_{l} \in \mathbb{N} \forall k \geq k_{l}, \eta_{x_{k l}-\alpha}(\varepsilon)>1-\lambda .
$$

In this case, one writes $\eta_{(e)}-\lim _{k, l} x_{k l}=\alpha$. Also, the element $\alpha$ is called the $\eta_{(e)}-$ limit of the double sequence $\left(x_{k l}\right)$.

Definition 3.7. Let $(D, \eta, *)$ be a PNS. A double sequence $\left(x_{k l}\right)$ is said to be statistically $e$-convergent to $\alpha \in D$ with respect to the probabilistic norm $\eta$ provided that for every $\varepsilon>0$ and $\lambda \in(0,1)$

has natural density 1 , that is

$$
\left\{l: \delta\left(\left\{k: \eta_{x_{k l}-\alpha}(\varepsilon) \leq 1-\lambda\right\}\right)=0\right\}
$$

$$
\delta\left(\left\{l: \delta\left(\left\{k: \eta_{x_{k l}-\alpha}(\varepsilon) \leq 1-\lambda\right\}\right)=0\right\}\right)=1 .
$$

In this case, one writes $s t_{\eta(e)}-\lim _{k, l} x_{k l}=\alpha$. Also, the element $\alpha$ is called the $s t_{\eta(e)}$-limit of the double sequence $\left(x_{k l}\right)$.

The following theorem gives the relation between $e$-convergence and statistical $e$-convergence on probabilistic normed spaces.

Lemma 3.8. Let $(D, \eta, *)$ be a PNS. Then, for every $\varepsilon>0, \alpha \in D$ and $\lambda \in(0,1)$ the following statements are equivalent:

i) $s t_{\eta(e)}-\lim _{k, l} x_{k l}=\alpha$.

ii) $\delta\left(\left\{l: \delta\left(\left\{k: \eta_{x_{k l}-\alpha}(\varepsilon) \leq 1-\lambda\right\}\right)=0\right\}\right)=1$.

iii) $s t_{(e)}-\lim _{k, l} \eta_{x_{k l}-\alpha}(\varepsilon)=1$.

Proof. From Definition 3.7, the first two parts are equivalent. $l \in L$,

(ii) $\Rightarrow$ (iii) Let $L=\left\{l: \delta\left(\left\{k: \eta_{x_{k l}-\alpha}(\varepsilon) \leq 1-\lambda\right\}\right)=0\right\}$. So $\delta(L)=1$. Then for all

$$
\left\{k:\left|\eta_{x_{k l}-\alpha}(\varepsilon)-1\right| \geq \lambda\right\} \subseteq\left\{k: \eta_{x_{k l}-\alpha}(\varepsilon) \geq 1+\lambda\right\} \cup\left\{k: \eta_{x_{k l}-\alpha}(\varepsilon) \leq 1-\lambda\right\} .
$$

So, we get for $l \in L$,

Then

$$
\delta\left(\left\{k:\left|\eta_{x_{k l}-\alpha}(\varepsilon)-1\right| \geq \lambda\right\}\right)=0 .
$$

which completes the proof.

$$
\delta\left(\left\{l: \delta\left(\left\{k:\left|\eta_{x_{k l}-\alpha}(\varepsilon)-1\right| \geq \lambda\right\}\right)=0\right\}\right)=1
$$


Theorem 3.9. Let $(D, \eta, *)$ be a PNS and let $\left(x_{k l}\right)$ be a double sequence whose terms are in the vector space D. If $\left(x_{k l}\right)$ is statistically e-convergent with respect to the probabilistic norm $\eta$ then its $s t_{\eta(e)}-$ limit is unique.

Proof. Suppose that there exist $\alpha$ and $\beta$ in $D$ with $\alpha \neq \beta$ such that $s t_{\eta(e)}-\lim _{k, l} x_{k l}=\alpha$ and $s t_{\eta(e)}-\lim _{k, l} x_{k l}=\beta$. Let $\xi>0$, choose $\lambda \in(0,1)$ such that

$$
(1-\lambda) *(1-\lambda) \geq(1-\xi)
$$

Let $\varepsilon>0$ be given. Then we define the following sets:

$$
\begin{aligned}
& L_{\eta, 1}(\lambda, \varepsilon):=\left\{l: \delta\left(\left\{k: \eta_{x_{k l}-\alpha}(\varepsilon) \leq 1-\lambda\right\}\right)=0\right\} \\
& L_{\eta, 2}(\lambda, \varepsilon): \quad=\left\{l: \delta\left(\left\{k: \eta_{x_{k l}-\beta}(\varepsilon) \leq 1-\lambda\right\}\right)=0\right\}
\end{aligned}
$$

and

$$
\begin{aligned}
K_{\eta, 1}(\lambda, \varepsilon) & : \quad=\left\{k: \eta_{x_{k l}-\alpha}(\varepsilon) \leq 1-\lambda\right\} \\
K_{\eta, 2}(\lambda, \varepsilon) & : \quad=\left\{k: \eta_{x_{k l}-\beta}(\varepsilon) \leq 1-\lambda\right\} .
\end{aligned}
$$

Since $s t_{\eta(e)}-\lim _{k, l} x_{k l}=\alpha$ and $s t_{\eta(e)}-\lim _{k, l} x_{k l}=\beta$ then we have $\delta\left(L_{\eta, 1}(\lambda, \varepsilon)\right)=1$, $\delta\left(L_{\eta, 2}(\lambda, \varepsilon)\right)=1, \delta\left(K_{\eta, 1}(\lambda, \varepsilon)\right)=0$ and $\delta\left(K_{\eta, 2}(\lambda, \varepsilon)\right)=0$, for all $\varepsilon>0$. Let

$$
\begin{aligned}
K_{\eta}(\lambda, \varepsilon) & =K_{\eta, 1}(\lambda, \varepsilon) \cap K_{\eta, 2}(\lambda, \varepsilon) \\
L_{\eta}(\lambda, \varepsilon) & =L_{\eta, 1}(\lambda, \varepsilon) \cap L_{\eta, 2}(\lambda, \varepsilon) .
\end{aligned}
$$

So we can see that $\delta\left(\mathbb{N} \backslash K_{\eta}(\lambda, \varepsilon)\right)=1$ and $\delta\left(\mathbb{N} \backslash L_{\eta}(\lambda, \varepsilon)\right)=0$. If $(k, l) \in\left(\mathbb{N} \backslash K_{\eta}(\lambda, \varepsilon)\right) \times\left(\mathbb{N} \backslash L_{\eta}(\lambda, \varepsilon)\right)$, then we have

$$
\eta_{\alpha-\beta}(\varepsilon) \geq \eta_{x_{k l}-\alpha}\left(\frac{\varepsilon}{2}\right) * \eta_{x_{k l}-\beta}\left(\frac{\varepsilon}{2}\right)>(1-\lambda) *(1-\lambda) \geq(1-\xi) .
$$

Since $\xi>0$ was arbitrary, we get $\eta_{\alpha-\beta}(\varepsilon)=1$ for all $\varepsilon>0$. So we get $\alpha=\beta$ from Definition 2.3 (ii). This completes the proof.

Theorem 3.10. Let $(D, \eta, *)$ be a PNS and let $\left(x_{k l}\right)$ be a double sequence whose terms are in the vector space $D$. If there exists $M=K \times L \subset \mathbb{N} \times \mathbb{N}$ such that $\delta(K)=1$ and $\delta(L)=1$ and $\eta_{(e)}-\lim _{(k, l) \in M} x_{k l}=\alpha$ then $s t_{\eta(e)}-\lim _{k, l} x_{k l}=\alpha$.

Proof. Suppose that there exists $M=K \times L$ such that $\delta(K)=1$ and $\delta(L)=1$ and $\eta_{(e)}-\lim _{(k, l) \in M} x_{k l}=\alpha$. Then for each $\varepsilon>0$ and $\lambda \in(0,1)$ there exists $l_{\varepsilon}$ such that for each $l \geq l_{\varepsilon}, l \in L$ there exists $k_{l}$ such that for each $k \geq k_{l}, k \in K$ we have $\eta_{x_{k l}-\alpha}(\varepsilon)>1-\lambda$. So for such $l$ we have

$$
\left\{k: \eta_{x_{k l}-\alpha}(\varepsilon) \leq 1-\lambda\right\} \subseteq \mathbb{N} \backslash\left\{K \backslash\left\{k_{1}, k_{2}, \ldots, k_{l}\right\}\right\} .
$$

Since $\delta(K)=1$ we have $\delta\left(\left\{k: \eta_{x_{k l}-\alpha}(\varepsilon) \leq 1-\lambda\right\}\right)=0$. On the other hand, this equation holds for each $l>l_{\varepsilon}, l \in L$. Therefore

$$
L \backslash\left\{l_{1}, l_{2}, \ldots, l_{\varepsilon}\right\} \subseteq\left\{l: \delta\left(\left\{k: \eta_{x_{k l}-\alpha}(\varepsilon) \leq 1-\lambda\right\}\right)=0\right\} .
$$

So we have

$$
\delta\left(\left\{l: \delta\left(\left\{k: \eta_{x_{k l}-\alpha}(\varepsilon) \leq 1-\lambda\right\}\right)=0\right\}\right)=1
$$

which completes the proof. 
So, if a double sequence $\left(x_{k l}\right)$ is $e$-convergent to $\alpha \in D$ with respect to the probabilistic norm $\eta$ then it is statistically $e$-convergent to $\alpha \in D$ on the PNS. But the converse of this implication may not be true. The following examples show that the converse of Theorem 3.10 does not hold in general.

Example 3.11. Let $(\mathbb{R},||$.$) be a real normed space and \eta_{x}(t)=\frac{t}{t+|x|}$ where $x \in \mathbb{R}$ and $t>0$. In this case $(\mathbb{R}, \eta,||$.$) is a PNS. Now we will give two examples in which our method of$ statistical $e$-convergence works but the other convergence methods do not work:

(i) Let $\left(x_{k l}\right)$ be defined as

$$
x_{k l}:=\left\{\begin{array}{cc}
k+l, & k \leq l, \\
k, & k>l \text { and } k \text { is square, } \\
0, & k>l \text { and } k \text { is not square. }
\end{array}\right.
$$

Then for every $\lambda \in(0,1)$ and for any $t>0$,

$$
\begin{aligned}
\left\{k: \eta_{x_{k l}}(t) \leq 1-\lambda\right\} & =\left\{k: \frac{t}{t+\left|x_{k l}\right|} \leq 1-\lambda\right\} \\
& =\left\{k:\left|x_{k l}\right| \geq \frac{\lambda t}{1-\lambda}>0\right\} .
\end{aligned}
$$

So we can get

$$
\delta\left(\left\{l: \delta\left(\left\{k: \eta_{x_{k l}}(t) \leq 1-\lambda\right\}\right)=0\right\}\right)=1 .
$$

Also it is easy to see that $\eta_{(e)}-\lim _{k, l} x_{k l}, \eta_{2}-\lim _{k, l} x_{k l}, s t_{2}-\lim _{k, l} x_{k l}$ and $s t_{\eta_{2}}-\lim _{k, l} x_{k l}$ do not exist. On the other hand, we can see from the above equality that $s t_{\eta(e)}-\lim _{k, l} x_{k l}=0$.

(ii) Let $\left(\alpha_{k l}\right)$ be defined as follows:

$$
\alpha_{k l}:= \begin{cases}k, & k \leq l, \\ 1, & k>l \text { and } k \text { is square, } \\ 0, & k>l \text { and } k \text { is not square. }\end{cases}
$$

Then we can see that $s t_{\eta(e)}-\lim _{k, l} \alpha_{k l}=0$. However $\eta_{(e)}-\lim _{k, l} \alpha_{k l}, \quad \eta_{2}-\lim _{k, l} \alpha_{k l}, s t_{2}-\lim _{k, l} \alpha_{k l}$ and $s t_{\eta_{2}}-\lim _{k, l} \alpha_{k l}$ do not exist.

Now we will show that the concept of statistical $e$-convergence of a double sequences on a PNS has some basic properties.

Lemma 3.12. Let $(D, \eta, *)$ be a PNS and let $\left(x_{k l}\right)$ and $\left(y_{k l}\right)$ be two double sequences on $D$.

(i) If $s t_{\eta(e)}-\lim _{k, l} x_{k l}=a$ and $s t_{\eta(e)}-\lim _{k, l} y_{k l}=b$, then $s t_{\eta(e)}-\lim _{k, l}\left(x_{k l}+y_{k l}\right)=a+b$.

(ii) If $s t_{\eta(e)}-\lim _{k, l} x_{k l}=a$ and $\alpha \in \mathbb{R}$, then $s t_{\eta(e)}-\lim _{k, l} \alpha \cdot x_{k l}=\alpha \cdot a$.

(iii) If $s t_{\eta(e)}-\lim _{k, l} x_{k l}=a$ and $s t_{\eta(e)}-\lim _{k, l} y_{k l}=b$, then $s t_{\eta(e)}-\lim _{k, l}\left(x_{k l}-y_{k l}\right)=a-b$.

Proof. (i) Let $s t_{\eta(e)}-\lim _{k, l} x_{k l}=a$ and $s t_{\eta(e)}-\lim _{k, l} y_{k l}=b, \varepsilon>0$ and $\xi \in(0,1)$. Choose $\lambda \in(0,1)$ such that $(1-\lambda) *(1-\lambda) \geq(1-\xi)$. Then we examine the following sets:

$$
\begin{aligned}
& L_{\eta, 1}(\lambda, \varepsilon): \quad=\left\{l: \delta\left(\left\{k: \eta_{x_{k l}-a}(\varepsilon) \leq 1-\lambda\right\}\right)=0\right\} \\
& L_{\eta, 2}(\lambda, \varepsilon):=\left\{l: \delta\left(\left\{k: \eta_{y_{k l}-b}(\varepsilon) \leq 1-\lambda\right\}\right)=0\right\}
\end{aligned}
$$

and

$$
\begin{aligned}
& K_{\eta, 1}(\lambda, \varepsilon): \quad=\left\{k: \eta_{x_{k l}-a}(\varepsilon) \leq 1-\lambda\right\} \\
& K_{\eta, 2}(\lambda, \varepsilon): \quad=\left\{k: \eta_{y_{k l}-b}(\varepsilon) \leq 1-\lambda\right\} .
\end{aligned}
$$


Since the double sequences $\left(x_{k l}\right)$ and $\left(y_{k l}\right)$ are statistically $e$-convergent to $a, b$, respectively then we have $\delta\left(K_{\eta, 1}(\lambda, \varepsilon)\right)=0, \delta\left(K_{\eta, 2}(\lambda, \varepsilon)\right)=0, \delta\left(L_{\eta, 1}(\lambda, \varepsilon)\right)=1$ and $\delta\left(L_{\eta, 2}(\lambda, \varepsilon)\right)=1$ for all $\varepsilon>0$. Now let

$$
\begin{aligned}
K_{\eta}(\lambda, \varepsilon) & =K_{\eta, 1}(\lambda, \varepsilon) \cap K_{\eta, 2}(\lambda, \varepsilon) \\
L_{\eta}(\lambda, \varepsilon) & =L_{\eta, 1}(\lambda, \varepsilon) \cap L_{\eta, 2}(\lambda, \varepsilon) .
\end{aligned}
$$

So, $\delta\left(\mathbb{N} \backslash K_{\eta}(\lambda, \varepsilon)\right)=1$ and $\delta\left(\mathbb{N} \backslash L_{\eta}(\lambda, \varepsilon)\right)=0$.

If $(k, l) \in\left(\mathbb{N} \backslash K_{\eta}(\lambda, \varepsilon)\right) \times\left(\mathbb{N} \backslash L_{\eta}(\lambda, \varepsilon)\right)$, then we have

$$
\begin{aligned}
\eta_{\left(x_{k l}+y_{k l}\right)-(a+b)}(\varepsilon) & =\eta_{\left(x_{k l}-a\right)+\left(y_{k l}-b\right)}(\varepsilon) \\
& \geq \eta_{x_{k l}-a}\left(\frac{\varepsilon}{2}\right) * \eta_{y_{k l}-b}\left(\frac{\varepsilon}{2}\right) \\
& >(1-\lambda) *(1-\lambda) \geq(1-\xi) .
\end{aligned}
$$

Then we see that

$$
\delta\left(\left\{k: \eta_{\left(x_{k l}-a\right)+\left(y_{k l}-b\right)}(\varepsilon) \leq 1-\xi\right\}\right)=0
$$

and

$$
\delta\left(\left\{l: \delta\left(\left\{k: \eta_{\left(x_{k l}-a\right)+\left(y_{k l}-b\right)}(\varepsilon) \leq 1-\xi\right\}\right)=0\right\}\right)=1
$$

so $s t_{\eta(e)}-\lim _{k, l}\left(x_{k l}+y_{k l}\right)=a+b$.

(ii) Case 1: Take $\alpha=0$ and let $s t_{\eta(e)}-\lim _{k, l} x_{k l}=a$. Let $\lambda \in(0,1)$ and $\varepsilon>0$. Then we can see that

$$
\eta_{0 \cdot x_{k l}-0 \cdot a}(\varepsilon)=\eta_{0}(\varepsilon)=1>1-\lambda .
$$

So we get

and

$$
\delta\left(\left\{k: \eta_{0 \cdot x_{k l}-0 \cdot a}(\varepsilon) \leq 1-\lambda\right\}\right)=\delta(\{\varnothing\})=0
$$

$$
\delta\left(\left\{l: \delta\left(\left\{k: \eta_{0 \cdot x_{k l}-0 \cdot a}(\varepsilon) \leq 1-\lambda\right\}\right)=0\right\}\right)=\delta(\mathbb{N})=1 .
$$

Hence we obtain $s t_{\eta(e)}-\lim _{k, l} 0 \cdot x_{k l}=0$.

Case 2: Take $\alpha \neq 0$. Since $s t_{\eta(e)}-\lim _{k, l} x_{k l}=a$, so for every $\varepsilon>0$ and $\lambda \in(0,1)$, we define the sets:

$$
L_{\eta}(\lambda, \varepsilon):=\left\{l: \delta\left(\left\{k: \eta_{x_{k l}-a}(\varepsilon) \leq 1-\lambda\right\}\right)=0\right\}
$$

and

$$
K_{\eta}(\lambda, \varepsilon):=\left\{k: \eta_{x_{k l}-a}(\varepsilon) \leq 1-\lambda\right\} .
$$

Then we see that $\delta\left(K_{\eta}(\lambda, \varepsilon)\right)=0$ and $\delta\left(L_{\eta}(\lambda, \varepsilon)\right)=1$. So $\delta\left(\mathbb{N} \backslash K_{\eta}(\lambda, \varepsilon)\right)=1$ and $\delta\left(\mathbb{N} \backslash L_{\eta}(\lambda, \varepsilon)\right)=0$. If $(k, l) \in\left(\mathbb{N} \backslash K_{\eta}(\lambda, \varepsilon)\right) \times\left(\mathbb{N} \backslash L_{\eta}(\lambda, \varepsilon)\right)$ then

$$
\begin{aligned}
\eta_{\alpha \cdot x_{k l}-\alpha \cdot a}(\varepsilon) & =\eta_{x_{k l}-a}\left(\frac{\varepsilon}{|\alpha|}\right) \\
& \geq \eta_{x_{k l}-a}(\varepsilon) * \eta_{0}\left(\frac{\varepsilon}{|\alpha|}-\varepsilon\right) \\
& =\eta_{x_{k l}-a}(\varepsilon) * 1 \\
& =\eta_{x_{k l}-a}(\varepsilon)>1-\lambda
\end{aligned}
$$

for $\alpha \in \mathbb{R}(\alpha \neq 0)$. So

$$
\delta\left(\left\{l: \delta\left(\left\{k: \eta_{\alpha \cdot x_{k l}-\alpha \cdot a}(\varepsilon) \leq 1-\lambda\right\}\right)=0\right\}\right)=1 .
$$

Hence we obtain $s t_{\eta(e)}-\lim _{k, l} \alpha \cdot x_{k l}=\alpha \cdot a$.

(iii) The proof of (iii) can be obtained from (i) and (ii). 


\section{References}

[1] Alsina, C., Schweizer, B., Sklar, A., Continuity properties of probabilistic norms, J. Math. Anal. Appl., 208(1997), 446-452.

[2] Boos, J., Classical and Modern Methods in Summability, Oxford University Press Inc., New York, 2000.

[3] Boos, J., Leiger, T., Zeller, K., Consistency theory for SM-methods, Acta Math. Hungar., 76(1997), 109-142.

[4] Fast, H., Sur la convergence statistique, Colloq. Math., 2(1951), 241-244.

[5] Frank, M. J., Probabilistic topological spaces, J. Math. Anal. Appl., 34(1971), 67-81.

[6] Fridy, J.A., On statistical convergence, Analysis (Berlin), 5(1985), 301-313.

[7] Guille'n, B.L., Lallena, J.A.R., Sempi, C., A study of boundedness in probabilistic normed spaces, J. Math. Anal. Appl., 232(1999), 183-196.

[8] Guille'n, B.L., Sempi, C., Probabilistic norms and convergence of random variables, J. Math. Anal. Appl., 280(2003), 9-16.

[9] Karakus, S., Statistical convergence on probabilistic normed spaces, Math. Commun., 12(2007), 11-23.

[10] Karakus, S., Demirci, K., Statistical convergence of double sequences on probabilistic normed space, Int. J. Math. and Math. Sci., Vol. 2007, Art. ID 14737, 11 pages (2007).

[11] Karakuş, S., Demirci, K., Duman, O., Equi-statistical convergence of positive linear operators, J. Math. Anal. Appl., 339(2008), 1065-1072.

[12] Karakus,, S., Demirci, K., Duman, O., Statistical convergence on intuitionistic fuzzy normed spaces, Chaos Solitons Fractals, 35(2008), 763-769.

[13] Menger, K., Statistical metrics, Proc. Natl. Acad. Sci. USA, 28(1942), 535-537.

[14] Moricz, F., Statistical convergence of multiple sequences, Arch. Math. (Basel), 81(2003), 82-89.

[15] Mursaleen, M., Edely, Osama H.H., Statistical convergence of double sequences, J. Math. Anal. Appl., 288(2003), 223-231.

[16] Pringsheim, A., Zur Theorie der zweifach unendlichen Zahlenfolgen, Math. Ann., 53(1900), 289-321.

[17] Schweizer, B., Sklar, A., Statistical metric spaces, Pacific J. Math., 10(1960), 313-334.

[18] Schweizer, B., Sklar, A., Probabilistic Metric Spaces, North Holland, New YorkAmsterdam-Oxford, 1983.

[19] Sever, Y., Talo, Ö., Statistical e-convergence of double sequences and its application to Korovkin type approximation theorem for functions of two variables, Iran J. Sci. Technol. Trans. A Sci., 41(2017), no. 3, 851-857.

[20] Sever, Y., Talo, Ö., On statistical e-convergence of double sequences, Iran J. Sci. Technol Trans A Sci., 42(2018), no. 4, 2063-2068.

[21] Steinhaus, H., Sur la convergence ordinaire et la convergence asymptotique, Colloq. Math., 2(1951), 73-74.

Sevda Akda $\breve{g}$

Sinop University, Faculty of Arts and Sciences, Department of Mathematics, 57000 Sinop, Turkey

e-mail: skarakus@sinop.edu.tr 